\title{
The floppy infants and metabolic causes
}

\author{
Sevil Dorum ${ }^{\oplus}$, Bayram Ali Dorum²® \\ ${ }^{1}$ Department of Pediatrics, Division of Metabolism, University of Health Sciences, Bursa Yüksek Ihtisas Training and Research Hospital, \\ Bursa, Turkey \\ ${ }^{2}$ Department of Pediatrics, Division of Neonatology, Bursa City Hospital, Bursa, Turkey
}

\section{ABSTRACT}

Objectives: To describe the clinical, metabolic and genetic characteristics of the floppy infants diagnosed at a tertiary care center.

Methods: A retrospective analysis was performed on the medical files of 90 floppy infants diagnosed in the pediatric metabolism department of our tertiary care center. Baseline descriptives, prenatal and perinatal data, results of genetic and metabolic tests as well as neuroradiological imaging findings were overviewed.

Results: Our series was comprised of 42 (46.7\%) females and 48 (53.3\%) males. Consanguineous marriages were detected in $60(66.7 \%)$ cases. There was no history of prenatal comorbidity or birth-related trauma or infections. Gestational age was $\geq 37$ weeks in $78(87 \%)$ infants. The average body weight was $2521.2 \pm 839.4$ grams (range: 1510-4300). The average height and head circumference were $48.9 \pm 0.9 \mathrm{~cm}$ and $33.9 \pm 0.7 \mathrm{~cm}$, respectively. The etiology of hypotonia was found to be central in $89(98.8 \%)$ infants. The most frequent diseases diagnosed were vitamin B12 deficiency (14.4\%), dystrophinopathy (7.7\%), spinal muscular atrophy $(5.5 \%)$, gangliosidosis $(3.3 \%)$, peroxisomal disease $(3.3 \%)$, Pompe disease $(3.3 \%)$, and Zellweger disease $(3.3 \%)$.

Conclusions: The floppy infant still constitutes a diagnostic challenge in spite of the technological advances in genetic, molecular and metabolic test methods. The priority and selection of diagnostic measures need to be determined on an individualized basis, and multidisciplinary and collaborative work is mandatory to set the diagnosis cost-effectively without delay. An algorithm based and individualized approach may provide a high diagnostic yield for the clinician.

Keywords: Floppy infant, hypotonia, newborn

$\mathrm{T}$ he floppy infant is characterized by congenital hypotonia, and it is a common disorder in the newborn period. It occurs as the subjective decrease of resistance to a passive range of motion in a newborn [1]. Some publications imply that the majority of hypotonic newborns are floppy due to central nervous system disorders; some hypotonic newborns have genetic diseases and metabolic disturbances, whereas the minority of hypotonic infants has neuromuscular and connective tissue disorders [2].
The clinical approach and investigative methods to be employed for the floppy infant had been well described in the medical literature [3-6]. Even though the identification of a neonate or infant lying in the supine position with a lack of power for spontaneous movements is easy, the appropriate route of diagnostic and investigative steps to be followed may pose a challenge. Before the popularization of DNA-based diagnostic studies, the diagnosis could be made using the identification of the specific syndromes that have cer- 
tain dysmorphic characteristics and clinical presentation. In some circumstances, invasive procedures such as electrophysiological studies or muscle biopsies may be necessary to rule in the diagnosis accurately. Moreover, molecular diagnostics and recognition of certain mutations linked with neuromuscular disorders facilitated the diagnostic processes for the floppy infant [35]. An appropriate diagnostic approach for the floppy infant involves selective use of available measures and integrative evaluation of data harvested from history, physical examination as well as the results of metabolic and genetic tests and radiologic imaging studies $[4,5]$. To effectively employ the advances in laboratory diagnosis and imaging, multidisciplinary evaluation by departments of genetics, pediatric neurology, pediatric metabolism, and pediatric neuroradiology is mandatory [6]. Careful clinical observation is vital to the proper evaluation of these critically ill children [7].

A better knowledge of the relative frequency of disorders that present with hypotonia may be helpful to select the appropriate investigations. Herein, we describe the perinatal and clinical features and to present the results of laboratory tests and imaging studies in the floppy infants. Therefore, we aimed to achieve an insight into the diagnostic profile of the floppy infants. We hope that our data may be beneficial to determine a stepwise algorithm in the diagnostic process for these patients.

\section{METHODS}

\section{Study Design}

This retrospective study was performed on data extracted from the medical files of 82 floppy infants diagnosed in the metabolism department of our tertiary care center between May 2017 and May 2018. Ethical approval for this study was obtained from Bursa Yüksek İhtisas Training and Research Hospital Clinical Research Ethics Committee (No: 2011KAEK-25/2018/11-04, Date: 07/11/2018) in accordance with the declaration of Helsinki-Ethical principles. All cases were infants younger than 12 months who underwent examination for generalized hypotonia, and neurologic, metabolic and genetic evaluation were carried out. Data were derived from the records of floppy infants and baseline descriptives, physical examination findings, prenatal and perinatal factors, diagnostic methods and results of imaging studies (computed tomography and magnetic resonance imaging [MRI]) were noted. The collection of data was performed after the written permission of hospital administration and the local ethics committee. Strict adherence to patient confidentiality was provided, and patients were randomly ordered by a computer program without the announcement of identity information.

\section{Our Clinical Approach}

In the first presentation of patients presenting with hypotonia, detailed history (prenatal, natal and postnatal history, neuromotor development steps, family history, similar story history, etc.) were taken. Height, body weight and head circumference were taken and a detailed physical examination was performed.

Complete blood count, biochemical tests (including glucose, alanine aminotransferase, aspartate aminotransferase, uric acid, creatine kinase, cholesterol etc.), thyroid function tests (free thyroxine and thyroid stimulating hormone), vitamin B12, folate, homocysteine, TANDEM-MS, blood gas analysis, ammonia and lactate tests were analyzed from all patients.

Blood amino acid chromatography, urine organic acids, very-long-chain fatty acids, and transferrin electrophoresis tests were performed as the second step in patients who could not be diagnosed by first step tests. Cranial MRI performed in patients who were considered necessary.

Patients who were required by the guidance of clinical and laboratory findings were consulted to the departments of pediatric neurology, ophthalmology, and pediatric cardiology for differential diagnosis.

The diagnosis that reached with these investigations, confirmed genetically. For the patients who could not be diagnosed, a clinical exome sequencing which is scanning 4900 genetic diseases, was performed.

\section{Statistical Analysis}

Data were analyzed using Statistical Package for Social Sciences (SPSS Inc., Chicago, IL, USA). Descriptive statistical methods were used and expressed as means, proportions, percentages and $95 \%$ confidence intervals. 
Table 1. Descriptive baseline variables

\begin{tabular}{|c|c|}
\hline Variables & $\begin{array}{c}\text { Data } \\
(n=90)\end{array}$ \\
\hline \multicolumn{2}{|l|}{ Gender } \\
\hline Female & $42(46.7 \%)$ \\
\hline Male & $48(53.3 \%)$ \\
\hline Consanguineous marriage & $60(66.7 \%)$ \\
\hline Prenatal morbidity & $0(0 \%)$ \\
\hline Birth trauma & $0(0 \%)$ \\
\hline \multicolumn{2}{|l|}{ Gestational age (weeks) } \\
\hline $33-36$ & $12(13 \%)$ \\
\hline Term & $78(87 \%)$ \\
\hline Birth weight (grams) & $2521.2 \pm 839.4$ \\
\hline Height (cm) & $48.9 \pm 0.9$ \\
\hline Head circumference (cm) & $33.9 \pm 0.7$ \\
\hline Admission age (min-max) & 7 days- 12 months \\
\hline \multicolumn{2}{|l|}{ Hypotonia } \\
\hline Peripheral & $1(1.2 \%)$ \\
\hline Central & $89(98.8 \%)$ \\
\hline Convulsion story & $19(21 \%)$ \\
\hline
\end{tabular}

Data was expressed as the mean \pm standard deviation or $\mathrm{n}(\%)$.

\section{RESULTS}

An overview of baseline descriptives is presented in Table 1. Of 90 patients, 42 (46.7\%) were females and $48(53.3 \%)$ were males. The admission ages of babies were between seven days and 12 months. Consanguineous marriages were detected in 60 $(66.7 \%)$ cases. There was no history of prenatal comorbidity or birth-related trauma or infections. Gestational age was $\geq 37$ weeks in $78(87 \%)$ infants. The average body weight was $2521.2 \pm 839.4$ grams (range: 700-4300). The average height and head circumference were $48.9 \pm 0.9 \mathrm{~cm}$ and $33.9 \pm 0.7 \mathrm{~cm}$, respectively. The etiology of hypotonia was found to be central in 89 (98.8\%) infants, whereas peripheral cause was determined in only $1(1.2 \%)$ case. There was a history of convulsion in $19(23.2 \%)$ of floppy infants in our series (Table 1).

Table 2 demonstrates the results of biochemical tests carried out in our patient population. Thyroid function tests, serum levels of vitamin B12, homocysteine, ammonium, CK, lactate, pyruvate, and uric acid were within normal range in the majority of our patients.

In Table 3, the results of metabolic tests carried out in selected patients were presented. Transferrin electrophoresis was performed to identify congenital disorders of glycosylation. One out of 16 patients revealed pathological results in transferrin electrophoresis. Very long chain fatty acid (VLCFA) levels were assessed to rule out peroxisomal diseases in 17 patients, and 2 of these infants yielded high levels of VLCFA. On electroencephalogram (EEG), 21 infants exhibited normal findings; whereas 19 infants had dysrhythmia. Magnetic resonance images were normal in 18 out of 43 patients. In contrary, in 25 patients had pathological changes such as thinning of the corpus callosum, cortical atrophy, cortical dysplasia, cerebral atrophy and glial changes in the cerebellum, pons and mesencephalon.

The most frequent diseases diagnosed were vitamin B12 deficiency (14.4\%), dystrophinopathy $(7.7 \%)$, spinal muscular atrophy (5.5\%),

Table 2. The results of biochemical tests

\begin{tabular}{lc}
\hline Variables & Data \\
\hline Thyroid function tests $(\mathbf{n}=\mathbf{9 0})$ & \\
$\quad$ Normal & $90(100)$ \\
\hline Vitamin $\mathbf{B}_{12}$ \& homocysteine $(\mathbf{n}=\mathbf{9 0})$ & \\
$\quad$ Normal & $77(85.6)$ \\
$\quad$ Low Vitamin $B_{12}$ level & $4(4.4)$ \\
$\quad$ Low vitamin $B_{12}$ level, high & $9(10)$ \\
homocysteine level & \\
Ammonium $(\mathbf{n}=\mathbf{9 0})$ & $87(96.7)$ \\
$\quad$ Normal & $3(3.3)$ \\
$\quad$ High & $82(91.1)$ \\
\hline Creatine kinase $(\mathbf{n}=\mathbf{9 0})$ & $8(8.9)$ \\
$\quad$ Normal & \\
\hline High & $88(97.8)$ \\
\hline Uric acid $(\mathbf{n}=\mathbf{9 0})$ & $2(2.2)$ \\
$\quad$ Normal & \\
\hline Low & $88(97.8)$ \\
\hline Lactate $(\mathbf{n}=\mathbf{9 0})$ & $2(2.2)$ \\
\hline Normal & $22(100)$ \\
\hline High & \\
\hline Pyruvate $(\mathbf{n}=\mathbf{2 2})$ & Normal \\
\hline
\end{tabular}


Table 3. The results of magnetic resonance imaging, electroencephalogram, metabolic tests, and clinical exome sequencing

\begin{tabular}{|c|c|}
\hline Variables & n (\%) \\
\hline \multicolumn{2}{|l|}{ Electroencephalogram $(n=40)$} \\
\hline Normal & $21(52.5)$ \\
\hline Dysrhythmic & $19(47.5)$ \\
\hline \multicolumn{2}{|l|}{ Magnetic resonance imaging $(n=43)$} \\
\hline Normal & $18(41.8)$ \\
\hline Pathological finding & $25(48.2)$ \\
\hline \multicolumn{2}{|l|}{ Transferrin electrophoresis $(n=16)$} \\
\hline Normal & $15(94)$ \\
\hline $\begin{array}{l}\text { Congenital Defect of Glycosylation } 2 \\
\text { (pattern 1) }\end{array}$ & $1(6)$ \\
\hline \multicolumn{2}{|l|}{ Very Long Chain Fatty Acids $(n=17)$} \\
\hline Normal & $15(88)$ \\
\hline High levels of c26 and c26:c22 & $2(12)$ \\
\hline \multicolumn{2}{|l|}{ Tandem $(n=90)$} \\
\hline Normal & $88(87.8)$ \\
\hline C3 carnitine levels $\uparrow$ & $1(1.1)$ \\
\hline C5DC carnitine levels $\uparrow$ & $1(1.1)$ \\
\hline \multicolumn{2}{|l|}{ Urine organic acids $(n=70)$} \\
\hline Normal & $60(85.7)$ \\
\hline $\begin{array}{l}\text { Urinary methylmalonic acid } \\
\text { levels } \uparrow\end{array}$ & $3(4)$ \\
\hline Urinary glutaric acid levels $\uparrow$ & $1(1.4)$ \\
\hline Oxoglutaric acid $\uparrow$ & $1(1.4)$ \\
\hline $\mathrm{N}$-acetylaspartate levels $\uparrow$ & $1(1.4)$ \\
\hline Oxoproline levels $\uparrow$ & $1(1.4)$ \\
\hline $\begin{array}{l}\text { 3-methylglutaconic acid, fumaric acid, } \\
\text { 3-OH isovaleric acid } \uparrow\end{array}$ & $2(3.3)$ \\
\hline Methylsitric acid $\uparrow$ & $1(1.4)$ \\
\hline \multicolumn{2}{|l|}{ Blood amino acid levels $(n=71)$} \\
\hline Normal & $66(93)$ \\
\hline Alanine levels $\uparrow$ & $2(2.8)$ \\
\hline Cystine levels $\downarrow$ & $2(2.8)$ \\
\hline Glycine $\uparrow$ & $1(1.4)$ \\
\hline \multicolumn{2}{|l|}{ Clinical exome sequencing $(n=69)$} \\
\hline Normal & $5(7.2)$ \\
\hline Diagnostic finding & $64(92.8)$ \\
\hline
\end{tabular}

Table 4. The final diagnosis of floppy infants

\begin{tabular}{|c|c|}
\hline Diagnosis & n (\%) \\
\hline Vitamin B12 deficiency & $13(14.4)$ \\
\hline Dystrophinopathy & $7(7.7)$ \\
\hline Spinal muscular atrophy & $5(5.5)$ \\
\hline Early infantile epileptic encephalopathy & $5(5.5)$ \\
\hline Pompe disease & $3(3.3)$ \\
\hline Gangliosidosis (GM1: 2 and GM2: 1) & $3(3.3)$ \\
\hline Peroxisomal disease & $3(3.3)$ \\
\hline Canavan disease & $2(2.2)$ \\
\hline Molybdenum cofactor deficiency A & $2(2.2)$ \\
\hline $\begin{array}{l}\text { Nieman Pick disease (Type A: } 1 \text { and } \\
\text { type C: } 1 \text { ) }\end{array}$ & $2(2.2)$ \\
\hline Leigh Syndrome & $2(2.2)$ \\
\hline Creatine transporter defect & $2(2.2)$ \\
\hline Glutaricaciduria (Type 2:1 and type 3:1) & $2(2.2)$ \\
\hline Congenital disorders of glycosylation & $2(2.2)$ \\
\hline Angelman syndrome & $2(2.2)$ \\
\hline Sandhoff disease & $1(1.1)$ \\
\hline EhlerDanlos syndrome & $1(1.1)$ \\
\hline Trichohepatoenteric syndrome & $1(1.1)$ \\
\hline Kabuki syndrome & $1(1.1)$ \\
\hline Tay Sachs disease & $1(1.1)$ \\
\hline $\begin{array}{l}\text { Leukoencephalopathy with vanishing } \\
\text { white matter }\end{array}$ & $1(1.1)$ \\
\hline Lissencephaly & $1(1.1)$ \\
\hline $\begin{array}{l}\text { Combined oxidative phosphorylation } \\
\text { deficiency type } 3\end{array}$ & $1(1.1)$ \\
\hline Metachromatic leukodystrophy & $1(1.1)$ \\
\hline Cat Cry syndrome & $1(1.1)$ \\
\hline Kleefstra syndrome & $1(1.1)$ \\
\hline I-cell disease & $1(1.1)$ \\
\hline Methylmalonicacidemia & $1(1.1)$ \\
\hline Walker Warburg syndrome & $1(1.1)$ \\
\hline Bohring Opitz syndrome & $1(1.1)$ \\
\hline Cornelia de Lange syndrome & $1(1.1)$ \\
\hline Mucopolysaccharidosis type $3 d$ & $1(1.1)$ \\
\hline Sepiapterinreductase deficiency & $1(1.1)$ \\
\hline Pyroglutamicacidemia & $1(1.1)$ \\
\hline Pyridoxine-dependent epilepsy & $1(1.1)$ \\
\hline $\begin{array}{l}\text { Biotin-thiamine-responsive basal } \\
\text { ganglia disease }\end{array}$ & $1(1.1)$ \\
\hline Undiagnosed & $14(15.5)$ \\
\hline
\end{tabular}


gangliosidosis (3.3\%), peroxisomal disease (3.3\%),Pompe disease (3.3\%), and Zellweger disease $(3.3 \%)$. Despite all these investigations, approximately $15 \%$ of the patients could not be diagnosed (Table 4 ).

\section{DISCUSSION}

The objective of the present study was to share the experience with the evaluation and diagnostic investigation of the floppy infant in our tertiary care center. Our retrospective series had a relatively large sample size; however, the applicability of our results to the general population necessitates implementation of further prospective, multi-centric trials on hypotonic infants. The main restrictions of the current study were retrospective design, missing information and lack of a control group for comparison. Furthermore, a standard set of tests cannot be administered to all infants routinely attributed to the unavailability of some molecular diagnostic tests within certain periods during this study.

We observed that the vast majority of the floppy infants revealed generalized hypotonia, which may be accompanied by additional symptoms such as convulsions, mental motor retardation. Congenital anomalies may be detected in a considerable proportion of infants. The gender distribution was fair. The lack of a control group limits the interpretation of the impacts of prenatal and perinatal factors on hypotonia. Genetic factors, family history for neuromuscular disorders as well as maternal diseases may affect the development of hypotonia [6]. Further trials are required for a better understanding of the combined effects of maternal, environmental and genetic factors in the pathogenesis of the floppy infants.

In previous reports, the advanced paternal age was supposed to be a confounding factor since it might increase the possibility of new dominantly inherited conditions [7]. However, we did not come across such a finding in our series. We noted that the majority of floppy infants were either term or near-term infants, who were appropriate for gestational age. A high rate of consanguineous marriages (66.7\%) was detected in our patient population, and this was one of our most remarkable outcomes. We suggest that discouragement of consanguineous marriages can be an important precaution to diminish the incidence of floppy infants. Tandem mass spectroscopy did not yield any abnormal results in the majority $(98.8 \%)$ of floppy infants. Similarly, blood amino acid levels were mostly within normal limits. On MRI views, findings such as thinning of the corpus callosum, cerebral or cortical atrophy and cortical dysplasia were detected. Electroencephalogram demonstrated multifocal epileptiform activity and cerebral dysfunction in some of our series. Clinical implications and the prognostic value of radiological, metabolic and genetic tests need to be elucidated in further trials. Serum levels of thyroid hormones, vitamin B12, homocysteine, ammonium, creatinine kinase, lactate, and pyruvate were generally within normal range. In conjunction with a report by Birdi et al. [3], central etiology was responsible for hypotonia in $98.8 \%$ of the floppy infants. Transferrin electrophoresis, which was used to identify the congenital disorders of glycosylation and evaluation for VLCFA, may be useful to identify the underlying condition in selected cases. Their priority and timing in the diagnostic study algorithm need to be well established since they are expensive and may not always be readily available.

The neuromuscular diseases were also diagnosed in our patient population. These disorders may be underdiagnosed, or they may be identified later following the onset of other specific symptoms else than hypotonia [8]. A proper sub-classification for floppy infants is essential to determine the risk and prognosis groups and employ the selective diagnostic modes. A small number of our patient population underwent MRI, and this was because CT scans were usually sufficient to identify the cranial abnormalities; thereby reducing the need for further MRI assessment. Integrative and combined use of radiological imaging studies in conjunction with genetic and metabolic tests may provide a highly sensitive and specific diagnostic tool for floppy infants. Advances in neuroimaging studies, as well as deoxyribonucleic acid (DNA)-based diagnostic tests, can improve the diagnostic accuracy and save time during diagnostic work. It has been recommended that considering these 2 options priorlycan improve cost-effectivity [9]. The indication and prognostic value for performance of other tests can be considered on an individualized basis. 3 Inborn 
metabolic errors and genetic diseases constitute a very wide spectrum, and clues such as multisystem involvement as well as typical dysmorphic features must be carefully overviewed before ruling in the diagnosis in floppy infants. Electrophysiological studies and muscle biopsy must be evaluated together for lower motor neuron disorders. It must be remembered that a good correlation between the experience and expertise of the clinician and laboratory is crucial [3]. David et al. [10] reported that there was a poor correlation between muscle biopsy results and electromyography findings. To overcome this diagnostic challenge, the combined use of muscle ultrasonography and electrophysiological studies can be useful [11].

The stepwise approach to the evaluation of a floppy newborn has already been proposed by Richer et al. [4]. Paro-Panjan et al. [1] have further divided this approach into six steps and proved that evaluation by the first five steps revealed the etiology in the majority of central hypotonia cases and $94 \%$ of all classifiable cases during the neonatal period. The diagnostic profile of the floppy infant is quite diverse. A good medical history, proper clinical observation, including neurologic examination, and the use of dysmorphology databases may aid in recognition of the majority of cases during the newborn period. The selective use of neuroimaging and genetic and biochemical methods contributed to the diagnosis in remaining cases. On the other hand, neurophysiologic investigations, specific molecular tests, and muscle biopsy may contribute to the diagnosis in minority of hypotonic newborns. Nevertheless, some cases including congenital myasthenic syndromes, some severe hypotonic forms of cerebral palsy, and syndromes of joint hyperlaxity are diagnosed only after close follow-up and frequent repetition of some neurophysiologic investigations [1].

To sum up, we determined that pathology was mainly central in the floppy infants and patients were usually appropriate for gestational age. In addition to metabolic and genetic diseases, connective tissue disorders must also be included in the differential diagnosis. Our results support the results of Birdi et al. [3], who suggested that clinical or whole exomesequence analysis, and neuroimaging studies should be considered first in infants with generalized hypotonia. If there is further need, more invasive test methods should be taken into account.

\section{Limitations}

The limitations of our study are that it is a retrospective study and that only hypotonic patients referred to the metabolism outpatient clinic were examined.

\section{CONCLUSION}

In conclusion, inborn errors of metabolism must be kept in mind in the differential diagnosis of the floppy infants. The priority and selection of diagnostic measures need to be determined on an individualized basis, and multidisciplinary and collaborative work is mandatory to set the diagnosis cost-effectively without delay. An algorithm based and individualized approach may provide a high diagnostic yield for the clinician.

\section{Conflict of interest}

The authors disclosed no conflict of interest during the preparation or publication of this manuscript.

\section{Financing}

The authors disclosed that they did not receive any grant during conduction or writing of this study.

\section{REFERENCES}

1. Paro-Panjan D, Neubauer D. Congenital hypotonia: is there an algorithm? Child Neurol 2004;19:439-42.

2. Thompson CE. Benign congenital hypotonia is not a diagnosis. Dev Med Child Neurol 2002;44:283-6.

3. Birdi K, Prasad AN, Prasad C, Chodirker B, Chudley AE. The floppy infant: retrospective analysis of clinical experience (1990 2000 ) in a tertiary care facility. J Child Neurol 2005;20:803-8.

4. Crawford TO.Clinical evaluation of the floppy infant. Pediatr Ann 1992;21:348-54.

5. Bergen BJ.Evaluation of the hypotonic or floppy infant. Minn Med 1985;68:341-7.

6. Prasad AN, Parasad C. Genetic evaluation of the floppy infant.Semin Fetal Neonatal Med 2011;16:99-108.

7. Richer LP, Shevell MI, Miller SP. Diagnostic profile of neonatal hypotonia: an 11-year study. Pediatr Neurol 2001;25:327.

8. McIntosh GC, Olshan AF, Baird PA. Paternal age and the risk of birth defects in offspring. Epidemiology 1995;6:282-8.

9. Mullaney P, Vajsar J, Smith R, Buncic JR. The natural history 
and ophthalmic involvement in childhood myasthenia gravis at the hospital for Sick Children. Ophthalmology 2000;107:504-10. 10. Bodensteiner JB, Byler DL, Jaynes ME. The utility of the determination of CTG trinucleotide repeat length in hypotonic infants. Semin Pediatr Neurol 1999;6:243-6.

11. David WS, Jones HR Jr. Electromyography and biopsy correlation with suggested protocol for evaluation of the floppy infant. Muscle Nerve 1994;17:424-30.

12. Aydinli N, Baslo B, Caliskan M, Ertas M, Ozmen M. Muscle ultrasonography and electromyography correlation for evaluation of floppy infants. Brain Dev 2003;25:22-4. 